Normal and hot electro-phonon resonance effect in a quasi-two-dimensional semiconductor system

This content has been downloaded from IOPscience. Please scroll down to see the full text. 1993 J. Phys.: Condens. Matter 52307

(http://iopscience.iop.org/0953-8984/5/15/004)

View the table of contents for this issue, or go to the journal homepage for more

Download details:

IP Address: 143.169.210.193

This content was downloaded on 09/10/2013 at 13:02

Please note that terms and conditions apply. 


\title{
Normal and hot electro-phonon resonance effect in a quasi-two-dimensional semiconductor system
}

\author{
W Xu, F M Peeters and J T Devreese $†$ \\ Departement Natuurkunde, Universiteit Antwerpen (UIA), Universiteitsplein 1, B-2610 \\ Antwerpen, Belgium
}

Received 20 July 1992, in final form 6 January 1993

\begin{abstract}
The electro-phonon resonance effect is a consequence of a resonant interaction between two electric subbands mediated by an optical phonon. It occurs in a quasi-twodimensional electron system each time the energy difference between two electric subbands equals the energy of a $w$ phonon. We study the influence of this effect on the electron mobility by using the momentum balance equation. The temperature and electron density dependences of the resonances are studied in the linear and non-linear response regimes.
\end{abstract}

\section{Introduction}

In a polar semiconductor at relatively high temperatures $(T>50 \mathrm{~K})$, the dominant factor limiting the mobility is the electron longitudinal optical (LO) phonon scattering process [1]. For electron motion in a quasi-two-dimensional system the effective electron-LO-phonon interaction is enhanced [2], which consequently leads to a lowering of the mobility. The quantum confinement in the $z$ direction implies that the electron momentum in the $z$ direction has no longer to be conserved during a scattering event. When several electric subbands are occupied in a quasi-two-dimensional electron gas (Q2DEG), resonant scattering between these levels will occur each time the energy difference of two electric subbands equals the energy of a LO phonon $\left(\hbar \omega_{L O}\right)$. These resonances are called electro-phonon resonances [3-9]. At resonance an enhancement of the scattering rate [8] will occur and the conductivity [8] and the mobility [9] will be suppressed.

With the development and application of the HEMT (high electron mobility transistor) structure [10], the study of this electro-phonon resonant effect is of importance in understanding transport phenomena in semiconductors. For electron motion in a twodimensional semiconductor system, the investigation of multi-subband transport effects at low temperature has received some attention $[4,9,11,12]$. In the present paper we are interested in effects that are the consequences of (i) the occupation of several electric subbands, and (ii) the electron-LO-phonon scattering process at a relatively high temperature.

The electro-phonon resonance effect is the electrical equivalent of the magnetic magnetophonon resonance effect, which was predicted by Gurevich and Firsov [13] (for a review see, for example, [3]). In the presence of a strong magnetic field, the magneto-phonon resonance effect occurs under the condition $N \hbar \omega_{c}=\hbar \omega_{L}$ where $\omega_{c}$ is the cyclotron 
frequency and $N=\left|n-n^{\prime}\right|$ is the difference between the Landau-level indices $n$ and $n^{\prime}$. This magneto-phonon resonance has been observed experimentally $[14,15]$ and studied theoretically [16] for a two-dimensional system. Much less research has been devoted to the electro-phonon resonance effect. Komiyama and co-workers [5] have shown that (i) the subband splitting in a MIS structure (i.e. a n-inversion layer on p-InSb) can be tuned electrically by varying the electron density, and (ii) the electro-phonon resonance effect can be observed when a strong quantizing magnetic field is applied perpendicular to the interface, which provides a 'zero-dimensional' energy level structure. A Monte Carlo simulation for high-field transport in a quantum wire was reported by Briggs and Leburton [6]. They found that the resonant inter-subband Lo phonon scattering occurs when the subband spacing is equal to the Lo phonon frequency. Recently, Kastalsky and co-workers [7] presented experimental evidence of the electro-phonon resonance effect in a $\mathrm{AlGaAs} / \mathrm{Al}_{x} \mathrm{Ga}_{1-x} \mathrm{As}$ triangular quantum well (TQW). We studied the linear electro-phonon resonance effects in [8] by calculating the electron-LO-phonon scattering rate and the conductivity as a function of the energy difference of the electric subbands with different confinement potentials: (i) a square well, (ii) a triangular well (to model the heterostructure), and (iii) a parabolic well. A relaxation time approximation method was introduced to calculate the conductivity.

In the present paper we employ the momentum balance equation proposed by Lei and Ting $[17,18]$ to study the electro-phonon resonance effects. One of the advantages of this approach is that dynamic electron-electron screening can be included when evaluating the electron transport characteristics. In section 2 the momentum balance equation for the mobility is derived by including electron scattered by LO phonons and electron-electron screening. The numerical results for the electro-phonon resonances in the mobility are presented in section 3 for linear and non-linear transport. Our conclusions are summarized in section 4.

\section{The momentum balance equation}

It has been demonstrated [19] that the momentum balance equation proposed by Lei and Ting $[17,18]$ provides a useful tool to study the transport properties of electrons in twodimensional systems. This momentum balance equation, which is based on the motion of the centre of mass of the electron system within the $2 \mathrm{D}$ plane, is physically equivalent to the momentum balance equation as derived from the Boltzmann equation which we applied to study warm-electron transport [20] and the electro-phonon resonances [9]. In most of the previous papers [17] on electron transport in a quasi-two dimensional system, one assumes that only the lowest electron subband is occupied. To observe the electro-phonon resonances we extend this balance equation to the situation of occupation of multi-subbands.

Recently [21] the applicability of the momentum balance equation, called the forcebalance equation, has been questioned. It is known that in the linear response regime such an approach leads to a resistivity $\rho=\langle 1 / \tau\rangle m / n_{\mathrm{e}} e^{2}$, when the correct result is given by $\rho=m /\langle\tau\rangle n_{\mathrm{e}} e^{2}$. For a degenerate electron distribution one has $\langle 1 / \tau\rangle=1 /\langle\tau\rangle$ at zero temperature and there is no discrepancy. However for a non-degenerate distribution [22] $\langle 1 / \tau\rangle$ and $1 /\langle\tau\rangle$ are different, where the difference may depend on temperature [23]. In spite of this criticism we will use the momentum balance equation because (i) we are dealing with a degenerate electron gas, although at non-zero temperature, (ii) in general it gives the correct qualitative behaviour, and (iii) it is relatively easy to obtain results in the non-linear regime. 
The Hamiltonian describing the general electron-phonon interaction in a many-electron quasi-two-dimensional system is given by

$$
H_{\text {cp }}=\sum_{\substack{n^{\prime}, n, j \\ q, q_{z}, \lambda}} M_{n^{\prime} n}\left(q, q_{z}, \lambda\right) \mathrm{e}^{\mathrm{i} q \cdot R_{(}}\left(b_{Q \lambda}+b_{-Q \lambda}^{\dagger}\right) \sum_{k, \sigma} c_{n^{\prime} k+q \sigma}^{\dagger} c_{n k \sigma}
$$

Following Lei and Ting [17] the momentum balance equation for the electron-phonon interaction is obtained by

$$
n_{\mathrm{e}} e \boldsymbol{E}+\boldsymbol{F}(\boldsymbol{v})=0
$$

with the force term

$$
\begin{aligned}
F(v)=2 \sum_{\substack{n^{\prime}, n \\
q, q_{s}, \lambda}} \mid & \left.M_{n^{\prime} n}^{0}\left(q, q_{z}, \lambda\right)\right|^{2} q \Pi^{0}\left(n^{\prime}, n, q, \Omega_{Q \lambda}+q \cdot v\right) \\
\times & {\left[n\left(\frac{\hbar \Omega_{Q \lambda}}{K_{\mathrm{B}} T}\right)-n\left(\frac{\hbar \Omega_{Q \lambda}+\hbar q \cdot v}{k_{\mathrm{B}} T_{\mathrm{e}}}\right)\right] }
\end{aligned}
$$

where $v$ is the electron velocity of the centre-of-mass motion, $T$ ( $T_{\mathrm{e}}$ ) the lattice (electron) temperature, $n$ the index of the electric subband, $\boldsymbol{Q}=\left(\boldsymbol{q}, q_{z}\right), \boldsymbol{q}=\left(q_{x}, q_{y}\right), \boldsymbol{R}=(x, y, z)$, $b_{Q \lambda}^{\dagger}$ and $b_{Q \lambda}$ are the phonon creation and annihilation operators, respectively, with wavevector $Q$ and frequency $\Omega_{Q \lambda}$ in branch $\lambda, c_{n k \sigma}^{\dagger}$ and $c_{n k \sigma}$ are the electron creation and annihilation operators, respectively, $n(x)=1 /[\exp (x)-1]$ is the Bose function, $\Pi^{0}\left(n^{\prime}, n, q, t\right)$ is the imaginary part of the electron density-density correlation function, and $M_{n^{\prime} n}^{0}\left(q, q_{z}, \lambda\right)=M_{n^{\prime} n}\left(q, q_{z}, \lambda\right) G_{n^{\prime} n}\left(q_{z}\right)$ the electron-phonon interaction matrix with the form matrix

$$
G_{n^{\prime} n}\left(q_{z}\right)=\int \mathrm{d} z \psi_{n^{\prime}}^{*}(z) \psi_{n}(z) \exp \left(-\mathrm{i} q_{z} z\right)
$$

which is determined by the electron wavefunction in the $z$ direction.

\subsection{Electron-LO-phonon coupling}

In case of electrons coupled to Lo phonons, (1) reduces to the Fröhlich Hamiltonian [24]

$$
H_{\text {ep }}=\sum Q_{Q}\left(V_{Q} a_{Q} \mathrm{e}^{\mathrm{i} q \cdot R}+V_{Q} a_{Q}^{\dagger} \mathrm{e}^{-\mathrm{i} q \cdot R}\right) \sum_{k \sigma} c_{n^{\prime} k+q \sigma}^{\dagger} c_{n k \sigma}
$$

where $V_{Q}=\mathrm{i}(4 \pi \alpha / V)^{1 / 2}\left(\hbar / 2 m^{*} \omega_{\mathrm{LO}}\right)^{1 / 4} \hbar \omega_{\mathrm{LO}} /\left(q^{2}+q_{z}^{2}\right)^{1 / 2}, \alpha$ is the electron-LO-phonon coupling constant ( $\alpha=0.068$ for GaAs), and $\omega_{\text {LO }}$ the LO phonon frequency ( $\hbar \omega_{\text {LO }}=$ $36.6 \mathrm{meV}$ for GaAs). The momentum balance equation (2) with an electric field applied along the $x$ direction is

$$
\begin{gathered}
n_{\mathrm{e}} e E+F\left(v_{x}\right)=0 \\
F\left(v_{x}\right)=2 \sum_{\substack{n^{\prime}, n_{2} \\
\boldsymbol{q}, q_{z}}}\left|v^{0}\left(\boldsymbol{q}, q_{z}\right)\right|^{2} q_{x} \Pi^{0}\left(n^{\prime}, n, q, \omega_{L O}+q_{x} v_{x}\right) \\
\times\left[n\left(\frac{\hbar \omega_{L O}}{k_{\mathrm{B}} T}\right)-n\left(\frac{\hbar \omega_{\text {LO }}+\hbar q_{x} v_{x}}{k_{\mathrm{B}} T_{\mathrm{e}}}\right)\right]
\end{gathered}
$$


with

$$
\left|v^{0}\left(q, q_{z}\right)\right|^{2}=\left(\frac{4 \pi \alpha}{V}\right) \sqrt{\frac{\hbar}{2 m^{*} \omega_{L} O}}\left(\hbar \omega_{L}\right)^{2} \frac{G_{n^{\prime} n}^{2}\left(q_{z}\right)}{q^{2}+q_{z}^{2}}
$$

where $v=v_{x} e_{x}$ is the average electron velocity, and $\Pi^{0}\left(n^{\prime}, n, q, \omega\right)$ is the Fourier transformation of the electron density-density correlation function which will be calculated in the following subsection.

In the limit of small electric fields, the electron response is described by the mobility $\mu$, which is given by

$$
\begin{aligned}
\frac{1}{\mu}=-\frac{2}{n_{\mathrm{e}} e v_{x}} & \sum_{\substack{n^{\prime}, n_{x} \\
q, q_{z}}}\left|v^{0}\left(\boldsymbol{q}, q_{\mathrm{z}}\right)\right|^{2} q_{x} \Pi^{0}\left(n^{\prime}, n, q, \omega_{\mathrm{LO}}+q_{x} v_{x}\right) \\
& \times\left[n\left(\frac{\hbar \omega_{\mathrm{LO}}}{k_{\mathrm{B}} T}\right)-n\left(\frac{\hbar \omega_{\mathrm{LO}}+\hbar q_{x} v_{x}}{k_{\mathrm{B}} T_{\mathrm{e}}}\right)\right] .
\end{aligned}
$$

\subsection{The electron density density correlation function}

The electron density-density correlation function plays a crucial role in the actual evaluation of electron transport:

$$
\Pi\left(n^{\prime}, n, q, \omega\right)=\Pi_{1}\left(n^{\prime}, n, q, \omega\right)+\mathrm{i} \Pi_{2}\left(n^{\prime}, n, q, \omega\right)
$$

with $\Pi_{1}\left(n^{\prime}, n, q, \omega\right)$ the real part, and $\Pi_{2}\left(n^{\prime}, n, q, \omega\right)$ the imaginary part, and thus

$$
\Pi^{0}\left(n^{\prime}, n, q, \omega\right)=\Pi_{2}\left(n^{\prime}, n, q, \omega\right) .
$$

For the situation where electron-electron screening is included within the random phase approximation (RPA) [25], we obtained for the imaginary part of the electron density-density correlation function

$$
\Pi^{0}\left(n^{\prime}, n, q, \omega\right)=\Pi^{0}(\beta, q, \omega)=\operatorname{Im}\left(\sum_{\gamma} \epsilon_{\beta \gamma}^{-1}(q, \omega) \Pi(\gamma, q, \omega)\right)
$$

where $\Pi\left(n^{\prime}, n, q, \omega\right)$ is the electron density-density correlation function in the absence of electron-electron screening, and the electron-electron screening is included through the dielectric function

$$
\epsilon_{\beta \gamma}(q, \omega)=\delta_{\beta \gamma}-V_{\beta \gamma}(q) \Pi(\gamma, q, \omega)
$$

where we used the notation $\beta, \gamma=\left(n^{\prime}, n\right)$, and

$$
V_{\beta \gamma}(q)=V_{m^{\prime} m n^{\prime} n}(q)=\frac{2 \pi e^{2}}{\kappa q} \int \mathrm{d} z_{1} \int \mathrm{d} z_{2} f_{\beta}\left(z_{1}\right) f_{\gamma}\left(z_{2}\right) \mathrm{e}^{-q\left|z_{1}-z_{2}\right|}
$$

is the Fourier-transformed Coulomb potential for electron scattering between subband $n$ and $n^{\prime}$ screened by the scattering between $m$ and $m^{\prime}$, with $f_{\beta}(z)=f_{n^{\prime} n}(z)=\psi_{n^{\prime}}^{*}(z) \psi_{n}(z)$ and $\kappa$ the static dielectric constant of the material ( $\alpha=12.9$ for GaAs). 
In the appendix we present a calculation of the imaginary part of the electron densitydensity correlation function when several electric subbands are included and in the presence of electron-electron screening.

In the absence of electron-electron screening the electron density-density correlation function takes the form

$$
\Pi\left(n^{\prime}, n, \boldsymbol{q}, \omega\right)=2 \sum_{k} \frac{f\left(E_{n^{\prime} k+q}\right)-f\left(E_{n k}\right)}{\hbar \omega+E_{n^{\prime} k+q}-E_{n k}+\mathrm{i} \delta}
$$

which gives

$$
\Pi_{1}\left(n^{\prime}, n, q, \omega\right)=2 P \sum_{k} \frac{f\left(E_{n^{\prime} k+q}\right)-f\left(E_{n k}\right)}{\hbar \omega+E_{n^{\prime} k+q}-E_{n k}}
$$

where $P$ stands for the principal value, and

$$
\Pi_{2}\left(n^{\prime}, n, \boldsymbol{q}, \omega\right)=-2 \pi \sum_{k}\left[f\left(E_{n^{\prime} k+q}\right)-f\left(E_{n k}\right)\right] \delta\left(\hbar \omega+E_{n^{\prime} k+q}-E_{n k}\right)
$$

where $E_{n k}=\hbar^{2} k^{2} / 2 m^{*}+\varepsilon_{n}$ is the electron energy with $\varepsilon_{n}$ the energy of the electric subband $n$, and $f(x)=1 /\left\{\exp \left[\left(x-\mu^{*}\right) / k_{\mathrm{B}} T_{\mathrm{e}}\right]+1\right\}$ is the Fermi-Dirac function with $\mu^{*}$ the chemical potential. After some simplifications we find

$$
\begin{aligned}
\Pi_{1}\left(n^{\prime}, n, \boldsymbol{q}, \omega\right) & =-\frac{1}{2 \pi} \frac{m^{*}}{\hbar^{2}} \frac{1}{\sqrt{\varepsilon_{q}}} \int_{0}^{1} \frac{\mathrm{d} x}{\sqrt{x}}\left(\sqrt{\varepsilon_{n^{\prime} n}-\varepsilon_{n}} f\left(-\left[\varepsilon_{n^{\prime} n}-\varepsilon_{n}\right] x+\varepsilon_{n^{\prime} n}\right)\right. \\
& \left.+\sqrt{\varepsilon_{n^{\prime} n}-\varepsilon_{n^{\prime}}-\hbar \omega} f\left(-\left[\varepsilon_{n^{\prime} n}-\varepsilon_{n^{\prime}}-\hbar \omega\right] x+\varepsilon_{n^{\prime} n}-\hbar \omega\right)\right)
\end{aligned}
$$

and

$\Pi_{2}\left(n^{\prime}, n, q, \omega\right)=-\frac{1}{2 \pi} \frac{m^{*}}{\hbar^{2}} \frac{1}{\sqrt{\epsilon_{q}}} \int_{0}^{\infty} \frac{d x}{\sqrt{x}}\left(f\left(x+\varepsilon_{n^{\prime} n}-\hbar \omega\right)-f\left(x+\varepsilon_{n^{\prime} n}\right)\right)$

with $\epsilon_{q}=\hbar^{2} q^{2} / 2 m^{*}$ and $\varepsilon_{n^{\prime} n}=\varepsilon_{n}+\left(\epsilon_{q}+\hbar \omega+\varepsilon_{n^{\prime}}-\varepsilon_{n}\right)^{2} / 4 \epsilon_{q}$.

\subsection{The momentum balance equation for the mobility}

With the above expressions for the electron density-density correlation function we can calculate the mobility:

$$
\begin{aligned}
\frac{1}{\mu}=-\frac{1}{n_{\mathrm{e}} e v_{x}} \frac{\alpha L_{0}}{\pi^{2}}\left(\hbar \omega_{\mathrm{LO}}\right)^{2} \sum_{\beta} \int_{0}^{\infty} \mathrm{d} q q^{2} X_{\beta}(q) \int_{-\pi}^{\pi} \mathrm{d} \theta \cos \theta \\
\times\left[n\left(\frac{\hbar \omega_{L} \mathrm{O}}{k_{\mathrm{B}} T}\right)-n\left(\frac{\hbar \omega}{k_{\mathrm{B}} T_{\mathrm{e}}}\right)\right] \operatorname{Im}\left[\sum_{\gamma} \epsilon_{\beta \gamma}^{-1}(q, \omega) \Pi(\gamma, q, \omega)\right]
\end{aligned}
$$

with $\omega=\omega_{\text {Lo }}+q v_{x} \cos \theta, L_{0}=\left(\hbar / 2 m^{*} \omega_{L O}\right)^{1 / 2}$ the unit of length $\left(L_{0}=39.5 \AA\right.$ for $\left.\mathrm{GaAs}\right)$, and

$$
X_{\beta}(q)=X_{n^{\prime} n}(q)=\int_{-\infty}^{\infty} \mathrm{d} q_{z} \frac{G_{n^{\prime} n}^{2}\left(q_{z}\right)}{q^{2}+q_{z}^{2}}
$$


The chemical potential $\mu^{*}$ is determined by electron number conservation:

$$
n_{\mathrm{e}}=\sum_{n} \int_{0}^{\infty} \mathrm{d} E \frac{D_{n}(E)}{\exp \left[\left(E-\mu^{*}\right) / k_{\mathrm{B}} T\right]+1}
$$

with $D_{n}(E)$ the density of states for electrons in subband $n$. Introducing the density of states (DOS) for a two-dimensional electron system

$$
D_{n}(E)=\frac{m^{*}}{\pi \hbar^{2}} \Theta\left(E-\varepsilon_{n}\right)
$$

equation (11a) reduces to

$$
n_{\mathrm{e}}=\frac{m^{*} k_{\mathrm{B}} T}{\pi \hbar^{2}} \sum_{n} \ln \left(1+\mathrm{e}^{-\left(\varepsilon_{n}-\mu^{*}\right) / k_{\mathrm{B}} T}\right) .
$$

In the case when a weak electric field is applied, the electron velocity is very small, which means $\left|q v_{x} \cos \theta\right| \ll 1$ and $T_{\mathrm{e}}=T$, and we obtain the ohmic mobility

$$
\begin{array}{r}
\frac{1}{\mu}=-\frac{\alpha L_{0} \hbar(\hbar \omega)^{2}}{\pi n_{\mathrm{c}} e k_{\mathrm{B}} T} N_{0}\left(N_{0}+1\right) \sum_{\beta} \int_{0}^{\infty} \mathrm{d} q q^{3} X_{\beta}(q) \\
\times \operatorname{Im}\left(\sum_{\gamma} \epsilon_{\beta \gamma}^{-1}\left(q, \omega_{\mathrm{LO}}\right) \Pi\left(\gamma, q, \omega_{\mathrm{LO}}\right)\right)
\end{array}
$$

with $N_{0}=1 /\left[\exp \left(\hbar \omega_{L} / k_{\mathrm{B}} T\right)-1\right]$ the LO phonon occupation number.

\subsection{The case of a square well}

To demonstrate the electro-phonon resonances, and for sake of simplicity, we consider the case of a quasi-two-dimensional electron system with a square-well ( $Q W)$ confinement of variable width. This has the advantages that (i) an arbitrary number of electric subbands can be included in the calcuiation, (ii) the position of the energy level of the subbands is tuned by changing the width of the quantum well, (iii) the electron density dependence of the electro-phonon resonance effect can be studied more directly, because the main effect of changing the electron density is to alter the chemical potential, and (iv) as will be shown in this subsection, the quantum-well confinement potential leads to a rather simple form for the form matrix and the electron-electron screening.

For the infinite-height square-well case, the eigenvalues $\varepsilon_{n}$ and envelope wavefunctions $\psi_{n}(z)$ are known analytically:

$$
\begin{aligned}
& \psi_{n}(z)=\sqrt{2 / L} \sin (n \pi z / L) \\
& \varepsilon_{n}=\frac{\hbar^{2}}{2 m^{*}} \frac{n^{2} \pi^{2}}{L^{2}}
\end{aligned}
$$

where $L$ is the width of the square well. In the above expressions we need

$$
X_{n^{\prime} n}(q)=\int_{-\infty}^{\infty} \mathrm{d} q_{z} \frac{G_{n^{\prime} n}^{2}\left(q_{z}\right)}{q^{2}+q_{z}^{2}}=\frac{\pi L}{16} I_{n^{\prime} n}(q)
$$


and

$$
V_{B Y}(q)=V_{m^{\prime} m n^{\prime} n}(q)=-\frac{\pi^{5} e^{2} L^{2} q}{8 \kappa}\left[1+(-1)^{m^{\prime}+m+n^{\prime}+n}\right] J_{m^{\prime} m n^{\prime} n}(q)
$$

where we introduce

$$
I_{n^{\prime} n}(q)=\frac{1}{a_{1}^{2}+c^{2}}+\frac{1+\delta_{n^{\prime} n}}{a_{2}^{2}+c^{2}}-\frac{\pi^{4} n^{\prime 2} n^{2} c}{\left.\left(a_{1}^{2}+c^{2}\right)^{2}\left(a_{2}^{2}+c^{2}\right)^{2}\right)}\left(1 \pm \mathrm{e}^{-2 c}\right)\left\{\begin{array}{l}
n^{\prime}+n=\text { odd } \\
n^{\prime}+n=\text { even }
\end{array}\right.
$$

and

$$
J_{m^{\prime} m n^{\prime} n}(q)=\frac{m^{\prime} m n^{\prime} n\left[1-(-1)^{n^{\prime}+n} \mathrm{e}^{-2 c}\right]}{\left(a_{1}^{2}+c^{2}\right)\left(a_{2}^{2}+c^{2}\right)\left(a_{3}^{2}+c^{2}\right)\left(a_{4}^{2}+c^{2}\right)}
$$

with $a_{1}=\pi\left(n^{\prime}+n\right) / 2, a_{2}=\pi\left(n^{\prime}-n\right) / 2, a_{3}=\pi\left(m^{\prime}+m\right) / 2, a_{4}=\pi\left(m^{\prime}-m\right) / 2$ and $c=q L / 2$. Notice that (14b) implies that, for the quantum well case, electron-electron screening occurs only when the condition $m^{\prime}+m+n^{\prime}+n=2 N$ (where $N$ is an integer) is satisfied. This greatly simplifies our calculation of the imaginary part of the electron density-density correlation function when we include electron-electron screening.

\section{Numerical results}

We perform the calculation for material parameters corresponding to GaAs: (i) the electron Lo phonon coupling constant $\alpha=0.068$, (ii) the effective mass ratio $m^{*} / m_{e}=0.0665$, (iii) the LO phonon energy $\hbar \omega_{L O}=36.6 \mathrm{meV}$, and (iv) the static dielectric constant $\kappa=12.9$. The LO phonon units are: the unit of length $L_{0}=\left[\hbar /\left(2 m^{*} \omega_{L O}\right)\right]^{1 / 2}\left(L_{0}=39.5 \AA\right.$ for GaAs), of velocity $v_{L O}=\left(2 \hbar \omega_{L O} / m^{*}\right)^{1 / 2}\left(v_{L O}=4.40 \times 10^{7} \mathrm{~cm} \mathrm{~s}^{-1}\right.$ for GaAs $)$, of temperature $T_{L O}=\hbar \omega_{L O} / k_{\mathrm{B}}\left(T_{\mathrm{LO}}=425 \mathrm{~K}\right.$ for $\left.\mathrm{GaAs}\right)$, and of electric field $E_{0}=\omega_{\text {LO }}\left(2 m^{*} \hbar \omega_{L O}\right)^{1 / 2} / e$ $\left(E_{0}=9.25 \times 10^{4} \mathrm{~V} \mathrm{~cm}^{-1}\right.$ for GaAs). In our numerical calculations we included the following electric subbands $n=1,2,3$ and 4 .

In figure 1 the chemical potential $\mu^{*}$ is shown (full curves) as a function of the well width for (a) different temperatures: $T=50,77,100,140$ and $220 \mathrm{~K}$ at a fixed electron density $n_{\mathrm{e}}=2 \times 10^{11} \mathrm{~cm}^{-2}$, and $(b)$ different electron densities: $n_{\mathrm{e}}\left(\times 10^{11} \mathrm{~cm}^{-2}\right)=0.1$, $0.8,2,5$ and 8 at a fixed temperature $T=77 \mathrm{~K}$. The chemical potential decreases with (i) increasing width of the quantum well, (ii) increasing lattice temperature, and (iii) decreasing electron density.

The electro-phonon resonance occurs at $L \sim\left(\left|n^{2}-n^{\prime 2}\right|\right)^{1 / 2} \pi L_{0}$, which is the condition that the energy difference between two subbands $n$ and $n^{\prime}$ equals a LO phonon energy $\hbar \omega_{L}$. The electro-phonon resonance effects are more pronounced when we plot the derivative of the mobility with respect to the width of the well. The ohmic mobilities along with their derivatives are shown in figure 2 as a function of the well width in the absence of e-e screening: (a) for different temperatures at a fixed electron density $n_{\mathrm{e}}=2 \times 10^{11} \mathrm{~cm}^{-2}$, and $(b)$ for different electron densities at a fixed temperature $T=77 \mathrm{~K}$. For illustrative purposes, and in order to be able to compare the results at different temperatures and electron densities, we scaled the mobility and its derivative to their values at $L=\pi L_{0}=124 \AA$. Note that (i) the strongest resonance is observed for $\varepsilon_{2}-\varepsilon_{1}=\hbar \omega_{\text {Lo }}$, which is reached at $L=\sqrt{3} \pi L_{0}=215 \AA$; (ii) the resonance that involves higher subbands is much weaker; (iii) the resonances are more pronounced for the case when the lowest subband $n=1$ is 


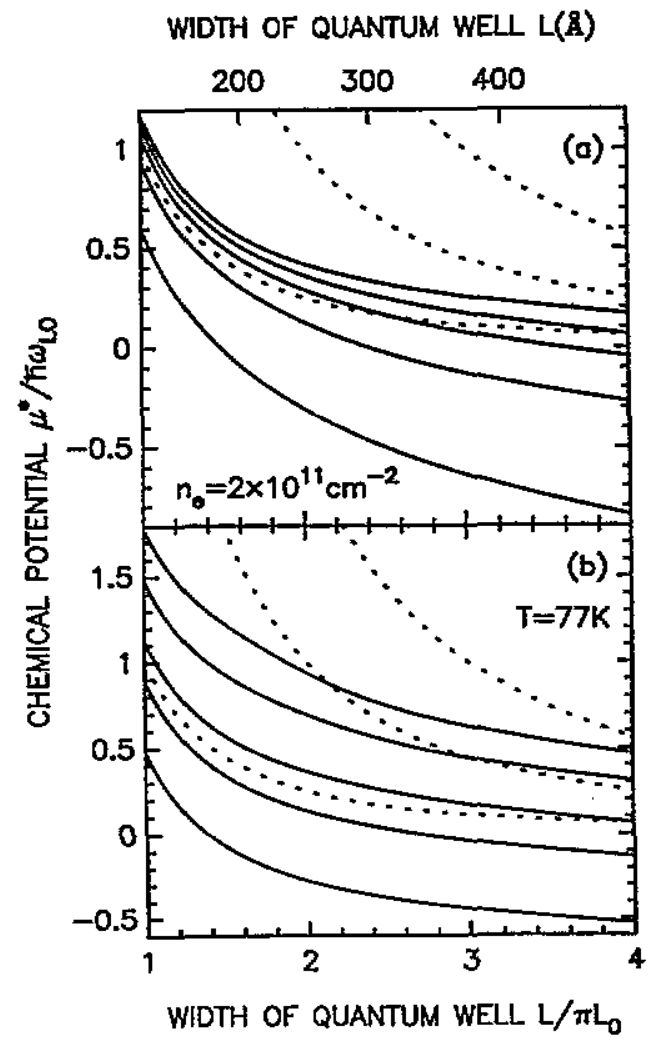

Figure 1. The chemical potential $\mu^{*}$ (full curves) and the energy levels (broken curves) of the different electric subbands as a function of the width of the quantum well for: (a) different temperatures at a fixed electron density $n_{e}=2 \times 10^{11} \mathrm{~cm}^{-2}$ : from top to bottom the full curves correspond to the temperature $T=50,77,100,140$ and $220 \mathrm{~K}$; and $(b)$ different electron densities at a fixed temperature $T=77 \mathrm{~K}$ : from top to bottom the full curves correspond to the electron density $n_{\mathrm{e}}\left(\times 10^{1 \mathrm{t}} \mathrm{cm}^{-2}\right)=8,5,2,0.8$ and 0.1 . The dotted curves correspond to the energy levels of the electric subbands: from top to bottom, $n=1,2$ and 3 . For GaAs the to phonon energy is $\hbar \omega_{2} O=36.6 \mathrm{meV}$ and the unit of length is $L_{0}=\sqrt{\hbar / 2 m^{*} \omega_{L O}}=39.5 \AA$.

involved; (iv) the amplitude of oscillations decreases quickly with increasing temperature, which is opposite to the temperature dependence found for magneto-phonon resonances; this is similar to what we found in [8] by using the relaxation time approach: the physical reason of this effect will be discussed below; (v) the amplitude of the oscillation decreases slightly with increasing electron density; and (vi) the resonances shift to smaller well widths with increasing temperature and increasing electron density.

To study the influence of electron-electron screening on the electro-phonon resonances, the ohmic mobility along with its derivative are shown in figure 3 (full curves) together with the previous results without screening (broken curves) as a function of the width of the quantum well $(a)$ for two different temperatures, and (b) for two different electron densities. Compared to the situation without e-e screening, we observe that the screening leads only to a small increase of the background for the mobility. This agrees with the results found by Lei [26] in a GaAs/AIGaAs heterojunction. The reason behind this is believed to be [26] that within RPA the enhancement of the LO phonon scattering rate due to dynamical effects almost compensates the reduction by static screening over the entire temperature and electron 

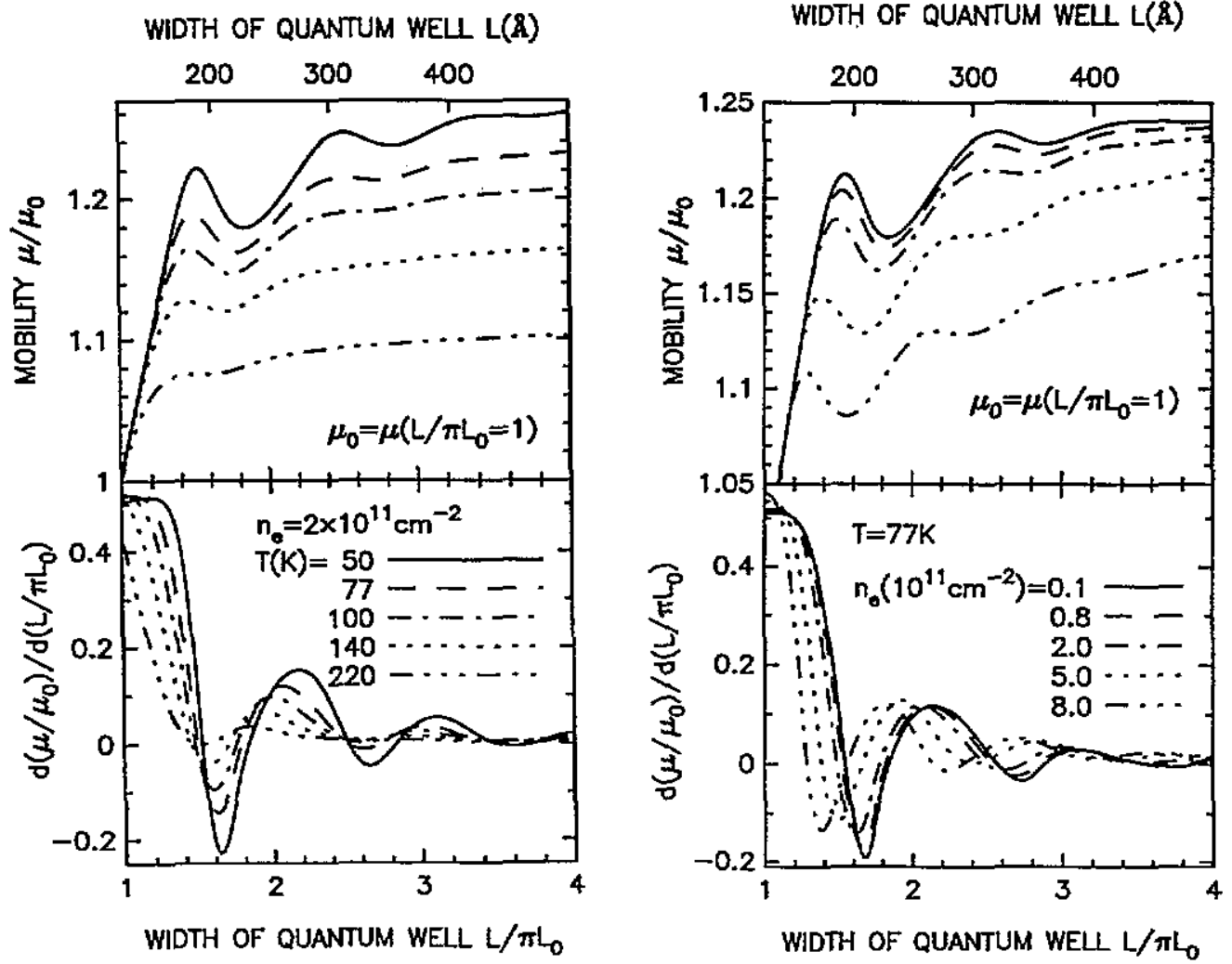

Figure 2. The ohmic mobility and its derivative as a function of the width of the quantum well in the absence of electron-electron screening. (a) Different temperatures at a fixed electron density $n_{\mathrm{c}}=2 \times 10^{11} \mathrm{~cm}^{-2}$. The mobilities are scaled to their values at $L=\pi L_{0}$ which are $\mu_{0}=\mu\left(L / \pi L_{0}=1\right.$ ) $=8605727$ (for $T=50 \mathrm{~K}$ ), 665279.7 (for $T=77 \mathrm{~K}$ ), 239385.8 (for $T=100 \mathrm{~K}$ ), 95613.13 (for $T=140 \mathrm{~K}$ ) and $43842.11 \mathrm{~cm}^{2} \mathrm{~V}^{-1} \mathrm{~s}^{-1}$ (for $T=220 \mathrm{~K}$ ). (b) Different electron densities at a temperature $T=77 \mathrm{~K}, \mu_{0}=\mu\left(L / \pi L_{0}=1\right)=669626.8$ (for $n_{\mathrm{e}}=10^{10} \mathrm{~cm}^{-2}$ ), 667962.7 (for $n_{\mathrm{e}}=8 \times 10^{10} \mathrm{~cm}^{-2}$ ), 665279.7 (for $n_{\mathrm{e}}=2 \times 10^{11} \mathrm{~cm}^{-2}$ ), 662460.8 (for $n_{e}=5 \times 10^{11} \mathrm{~cm}^{-2}$ ) and $677648.6 \mathrm{~cm}^{2} \mathrm{~V}^{-1} \mathrm{~s}^{-1}$ (for $n_{\mathrm{e}}=8 \times 10^{11} \mathrm{~cm}^{-2}$ ).

density region. Hence we conclude that the electron-electron screening does not effect the electro-phonon resonances very much for the case of Q2DEG in a square well. Therefore from now on (in order to limit CPU time) we will neglect electron-electron screening.

Non-linear effects can be studied in different ways: (i) we can heat up the electron system such that the electron temperature $\left(T_{e}\right)$ is larger than the lattice temperature, or (ii) the average electron velocity can be made non-zero by the application of a strong driving electric field. The effects of an electron temperature $T_{e}>T$ on the mobility can be evaluated by assuming (see (12)) that (i) the Lo phonon occupation number $N_{0}$ depends only on the lattice temperature, and (ii) the electron distribution depends on the electron temperature. The ohmic mobility along with its derivative is plotted as a function of the well width in figure 4 at a lattice temperature $T=4.2 \mathrm{~K}$ and at an electron density $n_{\mathrm{e}}=2 \times 10^{11} \mathrm{~cm}^{-2}$ for different electron temperatures. As far as the electro-phonon resonance is concemed, it is clear that changing the electron temperature leads to almost the same effect as changing the lattice temperature. This implies that the LO phonon emission scattering is the dominant process responsible for the electro-phonon resonances. 

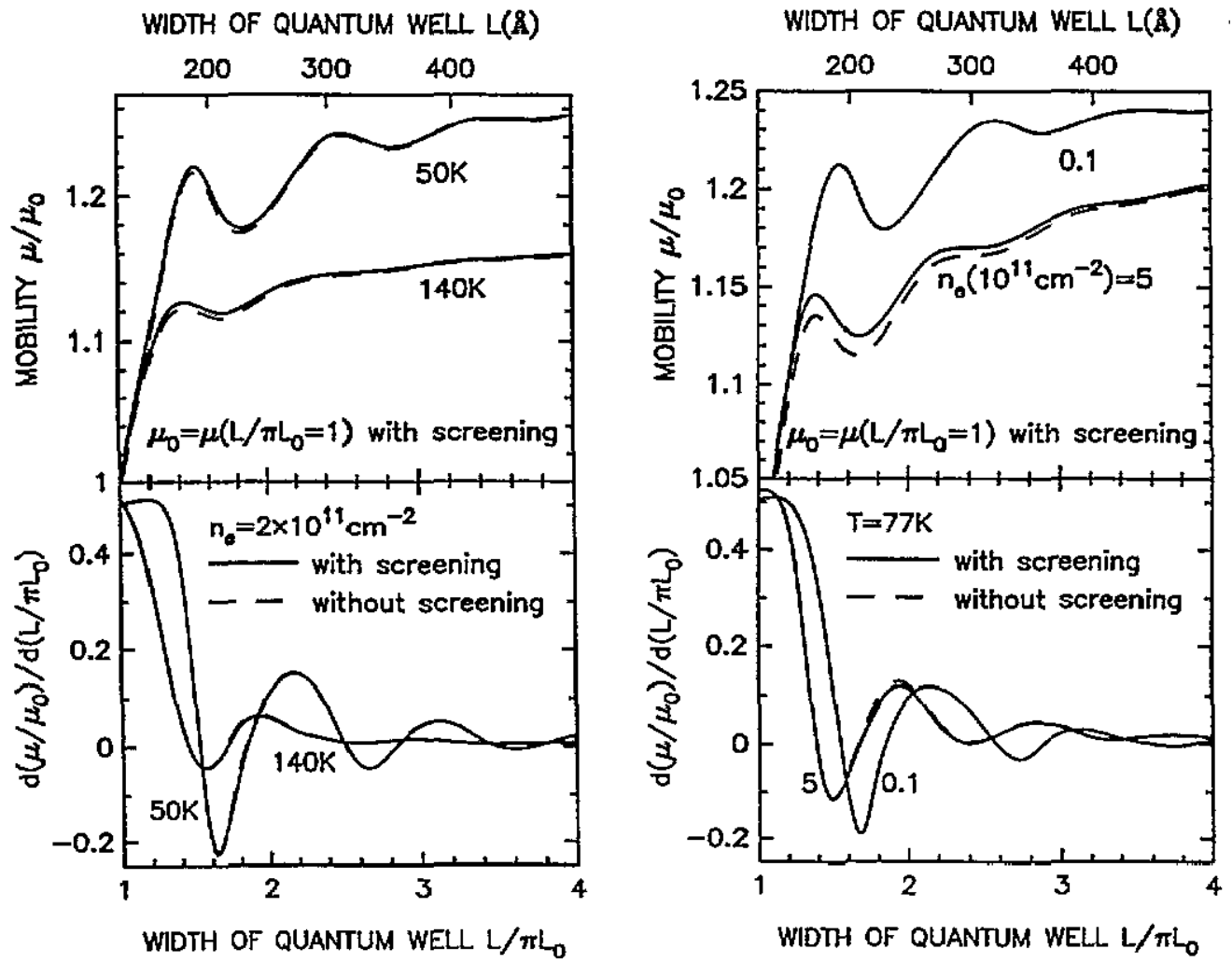

Figure 3. Influence of electron-electron screening (full curves) on the ohnic mobility and its derivative as a function of the width of the quantum well compared to the result in the absence of screening (broken curves) for $(a)$ different temperatures at electron density $n_{\mathrm{c}}=2 \times 10^{11} \mathrm{~cm}^{-2}$ : $\mu_{0}=\mu\left(L / \pi L_{0}=1\right.$ ) $=8642033$ (for $T=50 \mathrm{~K}$ ) and $96080.03 \mathrm{~cm}^{2} \mathrm{~V}^{-1} \mathrm{~s}^{-1}$ (for $T=140 \mathrm{~K}$ ); and $(b)$ different electron densities at temperature $T=77 \mathrm{~K}: \mu_{0}=\mu\left(L / \pi L_{0}=1\right)=669769.9$ (for $n_{\mathrm{e}}=10^{10} \mathrm{~cm}^{-2}$ ) and $670657.3 \mathrm{~cm}^{2} \mathrm{~V}^{-1} \mathrm{~s}^{-1}\left(\right.$ for $n_{\mathrm{e}}=5 \times 10^{11} \mathrm{~cm}^{-2}$ ).

The electro-phonon resonances are more pronounced at lower temperatures, which can be understood as follows: (i) with increasing temperature the Lo phonon absorption scattering rate is increased, which leads to an enhanced background scattering process and leads to a 'relative' smaller electro-phonon resonance; (ii) the electron's energy distribution is smoothed by thermal broadening at higher temperatures, which contributes to the smoothing of the resonances; and (iii) for the electro-phonon resonance effect the density of state (DOS) has only a step-like behaviour, which has to be compared with the singular nature of the DOS in the case of the magneto-phonon resonances. For the magneto-phonon resonances, thermal broadening is more important and consequently leads to a decreasing magneto-phonon resonance amplitude with increasing temperature.

So far the electro-phonon resonances have been studied within a linear response regime. Assuming that $T_{\mathrm{e}}=T$ the electro-phonon resonance effect for the mobility in the non-linear response regime (the hot-electron region) can be calculated within (10). In figure 5 the mobility and its derivative are plotted as a function of the width of the quantum well for different electron velocities at a temperature $T=77 \mathrm{~K}$ and an electron density $n_{\mathrm{e}}=2 \times 10^{11} \mathrm{~cm}^{-2}$. Note that (i) the electro-phonon resonances are more 


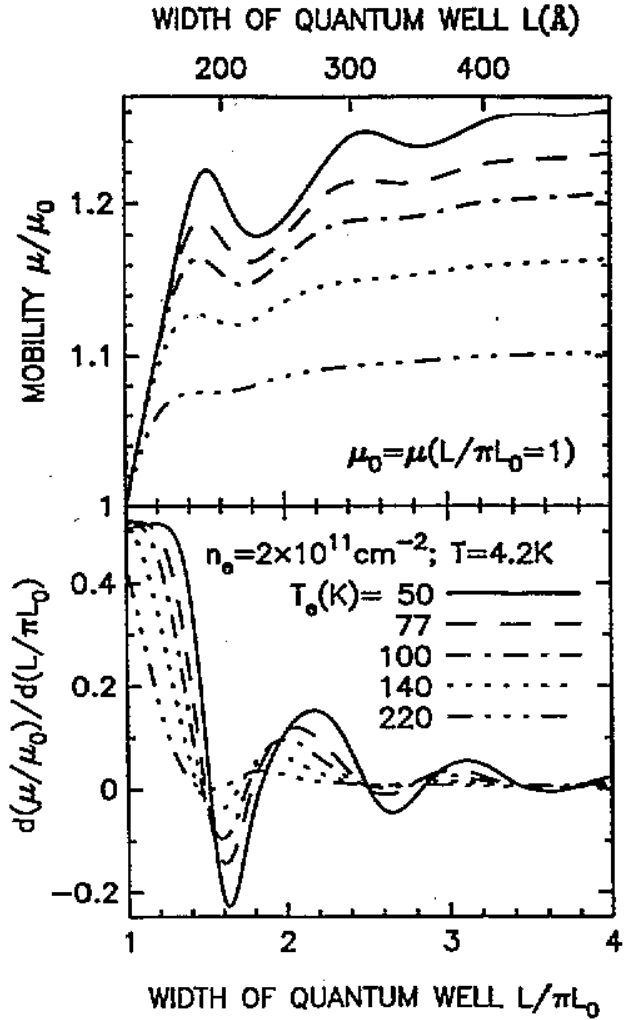

Figure 4. The ohmic mobility and its derivative as a function of the width of the quantum well for different electron temperatures $T_{e}$ at a fixed lattice temperature of $T=4.2 \mathrm{~K}$ and electron density $n_{\mathrm{e}}=2 \times 10^{11} \mathrm{~cm}^{-2}$.
WIDTH OF QUANTUM WELL L( $\$)$

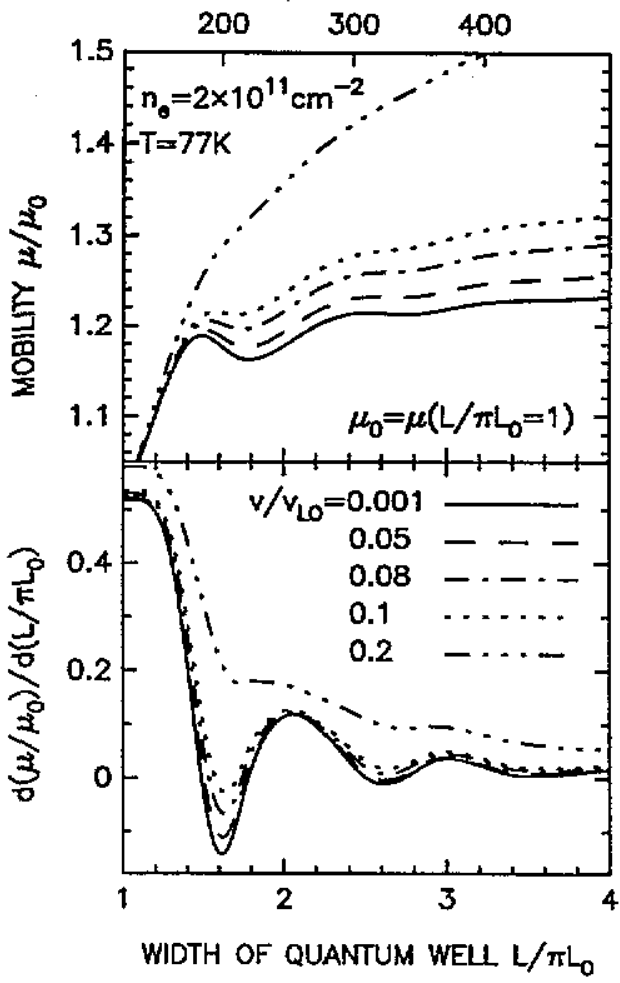

Figare 5. The mobility and its derivative in the nonlinear response regime as a function of the width of the quantum well at temperature $T=77 \mathrm{~K}$ and electron density $n_{e}=2 \times 10^{11} \mathrm{~cm}^{-2}$ for different electron velocities. For GaAs the unit of velocity is $v_{L O}=$ $\sqrt{2 \hbar \omega_{L O} / \mathrm{m}^{*}}=4.40 \times 10^{7} \mathrm{cms}^{-1} \cdot \mu_{0}=\mu\left(L / \pi L_{0}=\right.$ l) $=665261.8$ (for $v / v_{L O}=0.001$ ), 623751.0 (for $v / v_{L O}=0.05$ ), 565154.2 (for $v / v_{L O}=0.08$ ), 516968.5 (for $v / v_{L O}=0.1$ ) and $261793.4 \mathrm{~cm}^{2} \mathrm{~V}^{-1} \mathrm{~s}^{-1}$ (for $\left.v / v_{L}=0.2\right)$.

pronounced at small velocity (i.e. small electric field); (ii) the amplitude of the oscillations decreases with increasing electron velocity, and the effect disappears at the electron velocity $v=0.2 v_{\mathrm{L}}=8.8 \times 10^{6} \mathrm{~cm} \mathrm{~s}^{-1}$ for the case of $T=77 \mathrm{~K}$ and $n_{\mathrm{e}}=2 \times 10^{11} \mathrm{~cm}^{-2}$; and (iii) within the small-velocity regime, such that $v \leqslant 0.1 v_{\text {LO }}$ increasing the electron velocity leads only to a small change in the electro-phonon resonance effect.

\section{Summary}

In the present paper we have studied the electro-phonon resonance effect by using a momentum balance equation approach with the inclusion of the electron-LO-phonon interaction and electron-electron screening. The aim of this study was (i) to understand the transport phenomena in a quasi-two-dimensional semiconductor system; (ii) to give a 
quantitative description of electron transport in a Q2DEG in the situation where the electronLo-phonon coupling is the dominant scattering process limiting mobility and where several electric subbands are occupied by the electrons; (iii) to extend our previous study in order to include electron-electron screening; (iv) to study the electro-phonon resonance effect in the ohmic and hot-electron regime; and (v) to study the dependence on the lattice temperature, the electron temperature, the electron density and the electron average velocity of the electrophonon resonance effect.

Our conclusions of this study can be summarized as follows. (i) For a quasi-twodimensional electron system in a polar semiconductor such as GaAs and at relatively high temperatures the electro-phonon resonance effect occurs when the energy difference between two electric subbands equals a Lo phonon energy ( $\left.\hbar \omega_{L}\right)$.). (ii) The electro-phonon resonance effect is more pronounced at lower temperatures. With increasing temperature, the amplitude of the oscillations decreases and the position of the resonances shifts to smaller well widths in the case of a square-well confinement potential. (iii) With increasing electron density, the amplitude of the electro-phonon resonances slightly decreases and the position of the resonances shifts to smaller well widths. (iv) The strongest oscillation for the electronphonon resonances is observed for $\varepsilon_{2}-\varepsilon_{1}=\hbar \omega_{L}$. The resonances that involve higher subbands are much weaker. (v) Electron-electron screening does not greatly influence the resonance effect. Electron-electron screening increases slightly the background of the mobility. (vi) Increasing the electron temperature leads to almost the same effect as changing the lattice temperature for the electro-phonon resonances. The dominant scattering process that induces the resonances is $L O$ phonon emission. (vii) The electro-phonon resonances are more pronounced at low average electron velocity, i.e the weak electric field limit. The amplitude of the oscillations decreases with increasing electron velocity. (viii) We predict that the electro-phonon resonance is favourably observed in experiments done at relatively low temperatures, on a low electron density sample, and with a small electric field (the linear response regime).

In the present paper, numerical results were given only for the theoretical model of infinite-well confinement. Real systems have finite well depths or have a triangular-well-type of confinement, like the experimental systems of $[5,7]$. The present calculation can easily be generalized to such confinement potentials. The drawback of these more complicated confinement potentials is that only a very small number (i.e. one or two) electric subbands can be included in order to keep computer time at a realistic level. We believe that our conclusions concerning electron screening and the dependence of the electro-phonon resonances on the lattice and electron temperature, the electron density and the average electron velocity are valid irrespective of the exact form of the confinement potential.

It is now well established [28] that in real Q2D systems the bulk LO phonons are altered into interface and slab phonon modes. It is possible to generalize the present calculation in order to include these new type of phonons. Because the energy of the interface phonons are only slightly different from those of the bulk phonons the electro-phonon resonance condition will be practically the same. We do not expect very different results from such a generalization when compared to the present results. From [29] it may be inferred that the amplitude of the resonances can be slightly larger, because of the enhanced electron-phonon scattering due to scattering with the interface phonons.

\section{Acknowledgments}

One of us (FMP) is supported by the Belgian National Science Foundation. W Xu was supported by the Supercomputer Project of the NFWO (Nationaal Fonds voor 
Wetenschappelijk Onderzoek). This work is sponsored by Fonds voor Kollektief Fundamenteel Onderzoek (Belgium), project no. 2.0093.91, and by 'Diensten voor de Programmatie van het Wetenschapsbeleid' (Belgium) under contract no. IT/SC/24. FMP acknowledges discussion with A Kastalsky and J P Leburton. One of us (JTD) would like to thank R Evrard and J P Leburton for interesting discussions.

\section{Appendix}

In this Appendix we calculate the imaginary part of the electron density-density correlation with inclusion of the electron-electron screening as calculated within the random phase approximation (RPA) and where many electric subbands are present [27]. For convenience of presenting and deriving the formulae, we introduce the following notations. First, the correlation function in the absence of e-e screening:

$$
\Pi_{j}=\Pi_{j}^{\mathrm{R}}+\mathrm{i} \Pi_{j}^{\mathrm{I}}
$$

Second, the dielectric function in RPA:

$$
\epsilon_{i j}=\epsilon_{i j}^{\mathrm{R}}+\mathrm{i} \epsilon_{i j}^{\mathrm{I}} .
$$

Third, the correlation function in the presence of e-e screening:

$$
C_{i}=C_{i}^{\mathrm{R}}+\mathrm{i} C_{i}^{\mathrm{I}}=\epsilon_{i j}^{-1} \Pi_{j} .
$$

By solving the equations induced by the real and imaginary parts of (A3), we have for the imaginary part of the correlation function

$$
C_{i}^{\prime}=\left[\epsilon_{i j}^{\mathrm{R}}+\epsilon_{i j}^{\mathrm{X}}\left(\epsilon_{i j}^{\mathrm{R}}\right)^{-1} \epsilon_{i j}^{\mathrm{I}}\right]^{-1} l\left[\Pi_{j}^{\mathrm{X}}-\epsilon_{i j}^{\mathrm{I}}\left(\epsilon_{i j}^{\mathrm{R}}\right)^{-1} \Pi_{j}^{\mathrm{R}}\right] .
$$

After simplification we obtain for the imaginary part of the electron density-density correlation function

$$
C_{i}^{\mathrm{I}}=\left[\epsilon_{i j}^{\mathrm{R}}\left(\epsilon_{i j}^{\mathrm{I}}\right)^{-1}+\epsilon_{i j}^{\mathrm{I}}\left(\epsilon_{i j}^{\mathrm{R}}\right)^{-1}\right]^{-1}\left[\left(\epsilon_{i j}^{\mathrm{I}}\right)^{-1} \Pi_{j}^{\mathrm{I}}-\left(\epsilon_{i j}^{\mathrm{R}}\right)^{-1} \Pi_{j}^{\mathrm{R}}\right] .
$$

Retuming to our previous notations (see section 2) we have

$$
C_{i}^{\mathrm{I}}=\operatorname{Im}\left(\sum_{j} \epsilon_{i j}^{-1}(q, \omega) \Pi(j, q, \omega)\right)
$$

with

$$
\epsilon_{i j}^{\mathrm{R}}=\delta_{i j}-V_{i j}(q) \Pi_{1}(j, q, \omega) \quad \epsilon_{i j}^{\mathrm{I}}=-V_{i j}(q) \Pi_{2}(j, q, \omega)
$$

and

$$
\Pi_{j}^{R}=\Pi_{I}(j, q, \omega) \quad \Pi_{j}^{I}=\Pi_{2}(j, q, \omega) .
$$




\section{References}

[1] Seeger K 1973 Semiconductor Physics (Berlin: Springer)

[2] Peeters F M and Devreese J T 1987 Phys. Rev. B 364442

[3] Peeters F M and Devreese J T 1992 Semicond. Sci. Techrol. 715

[4] Stormer H L, Gossard A C and Wegmann W 1982. Solid State Commun. 41707

[5] Komiyama S, Eyferth H and Kotthaus J P 1980 J. Phys. Soc. Japan 49 suppl. A 687

[6] Briggs S and Leburton J P 1989 Superlath. Microstruc. 5145

Briggs S, Jovanovic D and Leburton J P 1989 Appl. Phys. Lett. 54 2012; 1989 Solid-State Electron. 321657

[7] Kastalsky A, Peeters F M, Chen W K, Florez L T and Harbison J P 1991 Appl. Phys. Lett. 591708

[8] Xu W, Peeters F M and Devreese J T 1993 Phys. Rev. B to be published

[9] Peeters F M, Kastalsky A, Xu W and Devreese J T 1990 20th Int. Conf. on the Physics of Semicond. (Thessaioniki, Greece, August 1990) ed. E M Anastassakis and J D Joannopoulos (Singapore: World Scientific) p 1138

[10] Mimura T, Hiyamizu S, Fujii T and Nanbu K 1980 Japan. J. Appl. Phys. 19 L225

[11] Mori S and Ando T 1980 J. Phys. Soc. Japan 48865

[12] Fletcher R, Zaremba E, D'lorio M, Foxon C T and Harris J J 1990 Phys. Rev. B 4110649

[13] Gurevich V L and Firsov Yu A 1963 Sov. Phys.-JETP 13137

[14] Nicholas R T 1985 Prog. Quantum Electr. 101

[15] Peterson R L 1975 Semiconductors and Semimetals ed. R K Willardson and A C Beer (New York: Academic)

[16] Warmenbol P, Peeters F M and Devreese J T 1988 Phys. Rev. B 374694

Mori N, Murata H, Taniguchi K and Hamaguchi C 1988 Phys. Rey. B 387622

[17] Lei X L and Ting C S 1984 Phys. Rev. B 30 4809; 1985 Phys. Rev. B 32 1112; 1987 Phys. Rev. B 368162 Lei X L, Birman J L and Ting C S 1985 J. Appl. Phys. 582270

[18] Cai W, Lei X L and Ting C S 1985 Phys. Rev. B 314070

[19] Magnus W, Sala C and De Meyer K 1991 Phys. Rev. B 439045

[20] Xn W, Peeters $F M$ and Devreese J T 1992 Semicond. Sci. Technol. 71251

[21] Argyres P N 1989 Phys. Rev. B 392982

Fishman R S and Mahan G D 1989 B 392990

[22] Huberman M and Chester G V 1975 Adv. Phys. 24489

[23] Peeters F M and Devreese J T 1983 Phys. Status Solidi (b) 115539

[24] Peeters F M and Devreese J T 1984 Solid State Phys. 3081

[25] Mahan G D 1981 Many-Particle Physics (New York: Plenum)

[26] Lei X L 1985 J. Phys. C: Solid State Phys. 18 L593

[27] Backes W H, Peeters F M. Brosens F and Devreese J T 1992 Phys. Rev. B 458437

[28] Wendier L 1985 Phys. Status Solidi(b) 129513

Mori N and Ando T 1989 Phys. Rev. B 406175

Hai G Q, Peeters F M and Devreese I T 1991 Phys. Rev. B 4211063

[29] Al-Dossary O, Babiker M and Constantinou N C 1992 Semicond. Sci. Technol. 791 